\title{
1 Typical retinotopic locations impact the time course of object coding
}

2

3 Daniel Kaiser, ${ }^{1, *}$, Merle M. Moeskops ${ }^{1}$, Radoslaw M. Cichy ${ }^{1,2,3}$

4

$5{ }^{1}$ Department of Education and Psychology, Freie Universität Berlin, Berlin, Germany

$6{ }^{2}$ Berlin School of Mind and Brain, Humboldt-Universität Berlin, Berlin, Germany

$7 \quad{ }^{3}$ Bernstein Center for Computational Neuroscience Berlin, Berlin, Germany

8

$9{ }^{*}$ Correspondence to:

10 Dr. Daniel Kaiser

11 Department of Education and Psychology

12 Freie Universität Berlin

13 Habelschwerdter Allee 45

1414195 Berlin, Germany

15 danielkaiser.net@gmail.com 


\section{Abstract}

17 In everyday visual environments, objects are non-uniformly distributed across visual

18 space. Many objects preferentially occupy particular retinotopic locations: for example,

19 lamps more often fall into the upper visual field, whereas carpets more often fall into the

20 lower visual field. The long-term experience with natural environments prompts the

21 hypothesis that the visual system is tuned to such retinotopic object locations. A key

22 prediction is that typically positioned objects should be coded more efficiently. To test

23 this prediction, we recorded electroencephalography (EEG) while participants viewed

24 briefly presented objects appearing in their typical locations (e.g., an airplane in the upper

25 visual field) or in atypical locations (e.g., an airplane in the lower visual field). Multivariate

26 pattern analysis applied to the EEG data revealed that object classification depended on

27 positional regularities: Objects were classified more accurately when positioned typically,

28 rather than atypically, already at $140 \mathrm{~ms}$, suggesting that relatively early stages of object

29 processing are tuned to typical retinotopic locations. Our results confirm the prediction

30 that long-term experience with objects occurring at specific locations leads to enhanced

31 perceptual processing when these objects appear in their typical locations. This may

32 indicate a neural mechanism for efficient natural scene processing, where a large number

33 of typically positioned objects needs to be processed.

\section{Keywords}

36 visual perception, object recognition, real-world regularities, location priors, EEG

37 decoding, multivariate pattern analysis 


\section{Introduction}

Visual objects are enclosed entities that can in principle be moved around freely. However, in everyday environments object positions are often quite constrained. For instance, consider the predictability in the locations of objects in a living room: The sofa is facing the TV, a table is in between the two, a lamp hangs from the ceiling, whereas carpets lie on the floor. This example illustrates that the object content of natural scenes is organized in repeatedly occurring positional structures (Bar, 2000; Chun, 2002). Many previous studies have investigated how inter-object relationships in these positional structures (e.g., lamps appearing above tables) impact behavioral performance and neural processing (Biederman, Mezzanotte, \& Rabinowitz, 1982; Kaiser, Stein, \& Peelen, 2014; Oliva \& Torralba, 2007; Wolfe, Võ, Evans, \& Greene, 2011). However, positional object structures often also imply that individual objects are associated with particular locations in space (e.g., lamps appearing in the upper part of a scene). It has recently been proposed that the visual system is tuned to these regularities (Kaiser \& Haselhuhn, 2017; Kravitz, Vinson, \& Baker, 2008), which could facilitate neural processing for individual objects appearing in retinotopic locations that correspond to their typical realworld locations.

Such location-specific variations in object coding are suggested by previous results that indicate the co-representation of object identity and location information in visual cortex: (1) cortical responses depend on the position of the object in the visual field (Hemond, Kanwisher, \& Op de Beeck, 2007; Hasson, Levy, Behrmann, Hendler, \& Malach, 2002), (2) object selective cortex contains information about both an object's identity and its location (Cichy, Chen, \& Haynes, 2011; Golomb \& Kanwisher, 2011; Hong, Yamins, Majaj, \& DiCarlo, 2017; Kravitz, Kriegeskorte, \& Baker, 2010; Schwarzlose, Swisher, Dang, \& 
62 Kanwisher, 2008), and (3) information about object identity and location emerge at

63 similar time points in visual processing (Isik, Meyers, Leibo, \& Poggio, 2014; Carlson,

64 Hogendoorn, Kanai, Mesik, \& Turret, 2011).

65 The link between identity and location information in object processing creates

66 the possibility that the two properties interact. In everyday environments, the visual

67 system is repeatedly faced with positional structures, where individual object positions

68 are highly predictable. Under typical viewing conditions, and unless directly fixated,

69 objects appearing in the upper part of scenes also more often occupy locations in the

70 upper visual field, while objects appearing in the lower part of scenes are repeatedly

71 encountered in the lower visual field. Through repeated exposure, retinotopic object-

72 coding mechanisms could get tuned to typical object locations in the visual field. Thus,

73 over time, neural channels are shaped to represent typical object-location conjunctions in

74 a maximally efficient way. These efficient location-specific object representations would

75 enhance the processing of an object when it appears in its typical locations within a scene

76 - and within the visual field. Evidence for such a processing enhancement has been found

77 in the domain of person perception, where typical configurations impact cortical

78 responses to individual face and body parts (Chan, Kravitz, Truong, Arizpe, \& Baker, 2010;

79 de Haas et al., 2016; Henriksson, Mur, \& Kriegeskorte, 2015). For example, in face-selective

80 visual cortex, response patterns are better discriminable for typically, as compared to

81 atypically, positioned face parts (de Haas et al., 2016), revealing visual processing

82 channels that are tuned to the spatial regularities in the face.

83 Here, we test the prediction that the positional regularities contained in natural

84 scenes can similarly facilitate the processing of everyday objects appearing in their typical

85 retinotopic locations. Participants viewed objects associated with upper and lower visual 
86 field locations (e.g., a lamp or a carpet) (Figure 1A) while we recorded

87 electroencephalography (EEG). We used multivariate classification on the EEG data

88 (Contini, Wardle, \& Carlson, 2017) to track the time course of object coding with high

89 temporal precision. Analyses revealed that after 140ms the visual processing of an object

90 is affected by its typical location in the visual field: Objects appearing in their typical

91 locations (e.g., a lamp in the upper visual field and a carpet in the lower visual field) could

92 be decoded more successfully than objects appearing in atypical locations (e.g., a carpet

93 in the upper visual field and a lamp in the lower visual field). These results suggest that

94 early stages of visual processing are tuned to the positional object structure of real-world

95 scenes. 
96

97

98

100

101

102

103

104

105

106

107

108

109

110

111

112

113

114

115

116

117

118

119

\section{Materials and Methods}

2.1 Participants

Thirty-four healthy adults (mean age 26.4 years, $S D=5.4 ; 23$ female) completed the experiment. The sample size was set a-priori, based on considerations regarding statistical power: A sample size of 34 is needed for detecting a simple effect with a medium effect size of $d=0.5$ with a probability of more than $80 \%$. All participants had normal or corrected-to-normal vision, provided informed consent and received monetary reimbursement or course credits for their participation. All procedures were approved by the ethical committee of the Department of Education and Psychology of the Freie Universität Berlin and were in accordance with the Declaration of Helsinki.

\subsection{Stimuli}

The stimulus set consisted of greyscale images of six objects associated with typical visual field locations, of which three were associated with upper visual field locations (lamp, airplane, and hat) and three were associated with lower visual field locations (carpet, boat, and shoe). For each object, ten exemplars were used (see Figure $1 \mathrm{~A}$ for stimulus examples). To avoid a confounding of visual field associations and other, conceptual stimulus attributes, the objects were matched for their categorical content (two furniture items, two transportation items, and two clothing items), so that high-level properties like the objects' size, manipulability and semantic associations were comparable across upper and lower visual field objects. To control for low-level visual factors, all stimulus images were gray-scaled and matched for overall luminance (using the SHINE toolbox; Willenbockel et al., 2010). Additionally, as a measure of low-level image similarity, pixelwise similarity was computed between all 60 images (i.e., 6 objects, 10 exemplars each), in a pairwise fashion: Pixel intensity values were correlated between all same visual field 
120 objects (e.g., lamps and airplanes) and between all different visual field objects (e.g.,

121 lamps and boats). No difference between all possible (600) within-field and all possible

122 (900) between-field correlations emerged, $t(1498)=0.50, p=0.62$, indicating that objects

123 with identical typical locations were no more similar in their low-level visual properties

124 than objects with different typical locations.

125 To ensure that the six objects could be reliably linked to a specific location, we

126 validated the association of the six objects with a specific part of the visual field in two

127 ways. First, we assessed the typical spatial distribution of each object in natural scenes,

128 assuming that natural scene photographs represent a snapshot of the visual field roughly

129 approximating natural viewing conditions. Hence, the distribution of the objects in the

130 scene image should be similar to their distribution across the visual field. To objectively

131 measure the typical position of each object within a scene, we queried a huge number of

132 scene photographs (>100,000) from the LabelMe toolbox, where human observers

133 annotated single objects by drawing labelled polygons (Russell, Torralba, Murphy, \&

134 Freeman, 2008). For all scenes containing a specific object we computed the mean pixel

135 coordinate of the area labeled as belonging to the object and then averaged these

136 positions across scenes. The resulting “typical” object locations showed that, as

137 expected, the upper visual field objects were associated with locations ( $y$ : vertical

138 coordinate from bottom (0) to top (1) of the scene) in the upper parts of scenes (lamp: $y$

$139=0.61, S D=0.17$; airplane: $y=0.52, S D=0.20$; hat: $y=0.60, S D=0.18$ ), while lower visual

140 field objects were associated with locations in the lower part of scenes (carpet: $y=0.17$,

$141 S D=0.13$; boat: $y=0.42, S D=0.18$; shoe: $y=0.37, S D=0.17)$. The typical location in scenes

142 differed significantly between objects associated with the upper and lower visual field, $t$ > 
$14311.4, p<.001$, for all pairwise comparisons. Figure $1 \mathrm{~B}$ shows the distribution of object

144 locations along the vertical axis of the scenes, split into 7 bins.

145 Second, we sought to demonstrate a correspondence between this automated,

146 scene-based measure and people's explicit associations of the objects with particular

147 locations in space. We thus asked a set of participants $(n=70$ for lamp/carpet, $n=60$ for

148 airplane/boat, $\mathrm{n}=65$ for hat/shoe; including the participants of the current study, after

149 the completion of the EEG experiment) to indicate the typical locations in which they

150 expect to see each of the six objects. In this task, participants were asked to drag the

151 image of a single exemplar of each object to its typical location on a computer screen

152 (imagining that the computer screen represents their field of view in a natural scene). The

153 central part of the screen - where the object initially appeared - was blocked (indicated

154 by a grey circle), so that participants (1) could not place the object in a central location of

155 the screen, and (2) had to move the object before proceeding. As expected, participants

156 more often chose upper screen positions ( $y$ : vertical coordinate from bottom (0) to top

157 (1) of the screen) for the upper visual field objects (lamp: $y=0.65, S D=0.19$; airplane: $y=$

$1580.67, S D=0.20$; hat: $y=0.57, S D=0.20$ ), and lower screen positions for the lower visual

159 field objects (carpet: $y=0.29, S D=0.22$; boat: $y=0.36, S D=0.18$; shoe: $y=0.30, S D=0.18$ ).

160 The vertical locations chosen by the participants differed significantly between objects

161 associated with the upper and lower visual field, $t>6.04, p<.001$, for all pairwise

162 comparisons. Figure $1 C$ shows the distribution of vertical object locations on the screen,

163 split into 7 bins. The scene-based measure and participants' explicit assessment thus

164 provided converging evidence for the association of the objects with specific spatial

165 locations. 
A

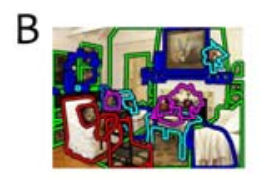

C

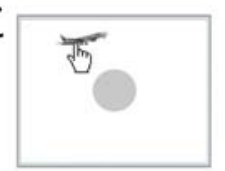

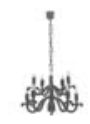
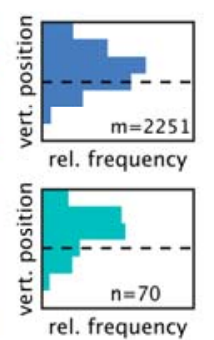
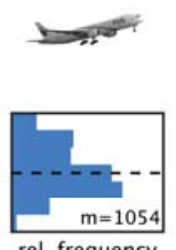

rel. frequency

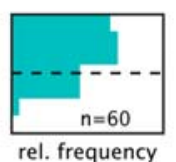

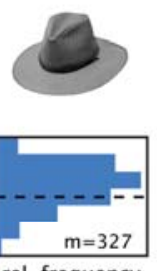

rel. frequency

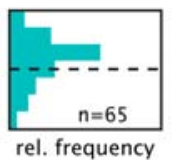

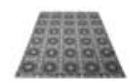

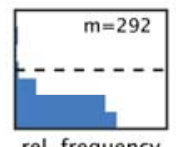

rel. frequency

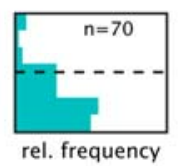

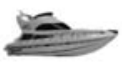

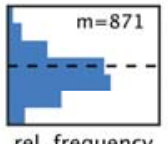

rel. frequency

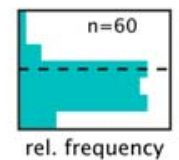

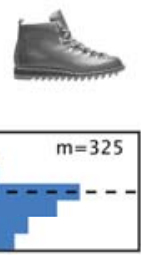

rel. frequency

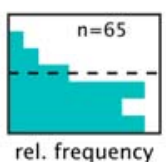

168 Figure 1. A) Example Stimuli. Ten different exemplar images of six objects each (here, one example image for each object shown) were used as stimuli, of which three objects were associated with the upper visual field (lamp, airplane, hat) and three were associated

171 with the lower visual field (carpet, boat, shoe). B) To validate our assessment of visual field associations, we automatically extracted the positions for each object in a large set

173 of labelled scene photographs taken from the LabelMe scene database (Russell et al.,

174 2008). For each scene, we determined the relative position of the object along the

175 vertical axis, and plotted the distribution across 7 bins ( $m$ : number of scenes for each

176 object). C) Additionally, we asked a group of participants to indicate for each object the

177 position that it typically occupies in the visual field by dragging the object to the desired

178 location. We then computed the distribution of relative locations along the vertical axis of

179 the screen, split into 7 bins (n: number of participants that indicated the typical location

180 for each object). Both measures confirmed the spatial priors associated with the six

181 objects. 
187 two locations for $150 \mathrm{~ms}$, followed by a variable inter-trial interval (randomly jittered, from

188 1250ms to $1750 \mathrm{~ms})$. The brief presentation time was chosen to not give participants

189 enough time to move their eyes towards the objects. Stimuli were presented at $3.25^{\circ}$

190 vertical eccentricity and subtended a visual angle of maximally $3^{\circ}$ in horizontal and vertical

191 axes. Stimulus presentation was controlled using the Psychtoolbox (Brainard, 1997).

192 Participants were asked to detect one-back repetitions on an object level (e.g., two

193 different airplanes in direct succession; see Figure $2 \mathrm{~A}$ ). Repetitions occurred on $13 \%$ of the

194 trials and equally often for typically and atypically positioned repetition targets and for

195 the top and bottom locations. Participants performed accurately on this task (mean

196 accuracy $96 \%, S D=2 \%$ ), with no difference in accuracy between typically and atypically

197 positioned objects, $t(33)=0.87, p=.391$. One-back repetition trials (i.e., the "second" trial

198 containing the repetition) were removed from all EEG analyses. The whole experiment

199 consisted of 1656 trials: in 1440 trials, each object exemplar was shown 12 times in each

200 location (i.e., 120 repetitions per object and location), while the remaining 216 trials were

201 one-back repetition trials. The experiment was split into 8 runs, and participants could

202 take breaks between the runs. Twelve participants completed an extended experimental

203 session with 2760 trials (including 360 repetition trials), which additionally contained the

204 same conditions at large eccentricities in half of the trials; these additional data are not

205 reported here. The 2400 non-repetition trials consisted of 10 repetitions of each object

206 exemplar in each of the four locations (i.e., 100 repetitions per object and location). The

207 extended experiment was split into 12 runs. 
209

A

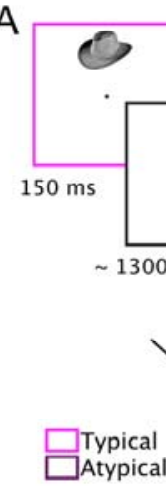

210

211

212

213

214

215

216

217

218

219

220

221

222

223

224

225

226

227

B

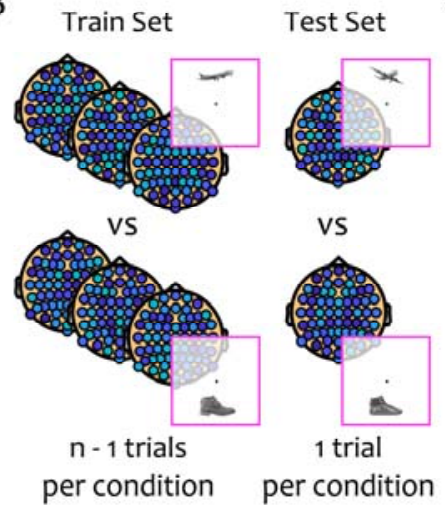

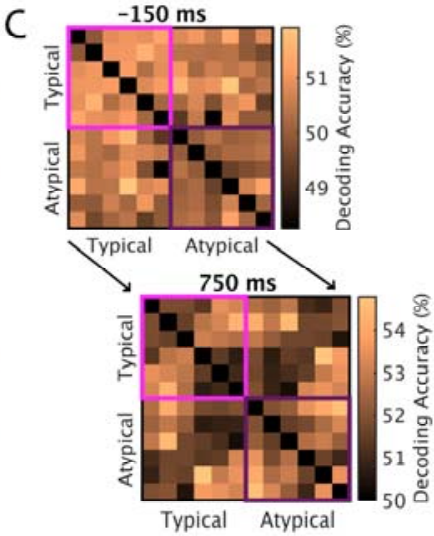

Figure 2. Paradigm and Classification Logic. A) Stimuli were presented for $150 \mathrm{~ms}$ in upper

or lower visual field locations, corresponding to an object's typical or an atypical location.

Participants were instructed to detect occasional one-back repetitions on an object-level

(e.g., two airplanes in a row; irrespective of the stimulus location) by pressing a button.

Colors indicating the two regularity conditions are shown for illustrative purposes only. B)

Multivariate classification was performed on response patterns across all electrodes,

separately for each pairwise combination of objects (exemplified here by airplane and

shoe in regular locations). The data was split into two sets: a training set consisting of all

(but one) trials for each object and a testing set consisting of the two left-out trials. LDA

classifiers were repeatedly trained and tested until every trial was left out once and

accuracy was averaged across these repetitions. C) The pairwise classification analysis

was repeated for each $10 \mathrm{~ms}$ time bin, resulting in a 12-by-12 matrix of pairwise

classification accuracies (with an empty diagonal) at every time point. To determine

differences between typically and atypically positioned objects, pairwise comparisons

within the typically placed objects (pink rectangle, upper left) and within the atypically

placed objects (purple rectangle, lower right) were averaged and compared (Figure 3). 
234 (Oostenveld, Fries, Maris, \& Schoffelen, 2011). The continuous EEG data was epoched into

235 trials ranging from $150 \mathrm{~ms}$ before stimulus onset to $750 \mathrm{~ms}$ after stimulus onset. Trials

analyses. Blink and eye movement artifacts were identified and removed using

Tovar, Alink, \& Kriegeskorte, 2013), the data was downsampled to $100 \mathrm{~Hz}$.

245 resolution. The analysis was performed pairwise, for all possible combinations of the six

247 always trained and tested on data from two conditions (e.g., an airplane in the upper

248 visual field versus a carpet in the lower visual field), using a leave-one-out partitioning

249 scheme (Figure 2B). The training set consisted of all but one trials for each of the two

250 conditions, while one trial for each of the two conditions was held back and used for

251 classifier testing. This procedure was repeated until every trial was left out once. Classifier 
252 performance was averaged across these repetitions. The pairwise decoding analysis

253 resulted in 12-by-12 matrix of decoding accuracies at each time point (reflecting all

254 comparisons between the six objects appearing in the two locations) (Figure 2C).

255

256

257

258

259

260

261

262

263

264

265

266

267

268

269

270

271

272

273

274

275

\subsection{Overall classification dynamics}

To assess the overall classification dynamics over time, we computed the general discriminability of the twelve different conditions. All pairwise classification accuracies were averaged, revealing a time course of object decoding independently of the positional regularities. This time course of overall classification accuracy was used to define time points of interest at the peaks of the classification time series, where classification performance was particularly pronounced. Using a "region of interest" logic frequently applied in fMRI analyses (Poldrack, 2007), we used these peaks as "time points of interest" to increase the detection power of subsequent analyses.

\subsection{Object classification in typical and atypical locations}

To determine an effect of positional regularity on object decoding, we compared performance when classifying among typically positioned objects versus among atypically positioned objects. Pairwise classification accuracies were averaged for all comparisons between typically positioned objects (e.g., an airplane in the upper visual field versus a shoe in the lower visual field) and for all comparisons between atypically positioned objects (e.g., a shoe in the upper visual field versus an airplane in the lower visual field) (Figure $2 \mathrm{C}$ ). Subsequently, the two resulting classification time series (for typically and atypically positioned objects) were compared. To increase the statistical power of this comparison, we specifically focused on the effect of positional regularity at the peaks in overall classification.

\subsection{Sensor-space searchlight analysis}


277 performed a sensor-space searchlight analysis. For this analysis, the pairwise classification

278 procedure was repeated for neighborhoods of seven adjacent electrodes around each

279 individual electrode; the resulting classification accuracy was then mapped onto a scalp

280 representation. This procedure allowed us to infer the approximate spatial distribution of

281 classification differences between typically and atypically positioned objects. As for one

282 participant only data from 32 electrodes was available, this participant was not included

283 in the searchlight analysis.

\subsection{Statistical testing}

across time we used a threshold-free cluster-enhancement procedure (Smith \& Nichols,

295 information Bayes factors (BF) were calculated as an additional measure of evidential 


\section{$298 \quad 3$ Results}

In a first step, we characterized the overall response dynamics observed in the

301 pairwise classification analysis, which allowed us to restrict subsequent analyses to time

302 points where classification performance was particularly pronounced. For this, we

303 computed an overall measure of pairwise classification by averaging across all unique off-

304 diagonal elements of the pairwise classification matrices (Figure $2 \mathrm{C}$ ), resulting in a single

305 classification time series. This analysis revealed robust above-chance classification

306 starting from $70 \mathrm{~ms}$ after stimulus onset and prominently peaking at $140 \mathrm{~ms}$ and $220 \mathrm{~ms}$

307 (Figure 3A). These two clear peaks in the classification time series were used as time

308 points of interest for subsequent analyses, as we reasoned that differences between

309 typically and atypically positioned objects would be most pronounced at time points at

310 which objects were most discriminable. 
A

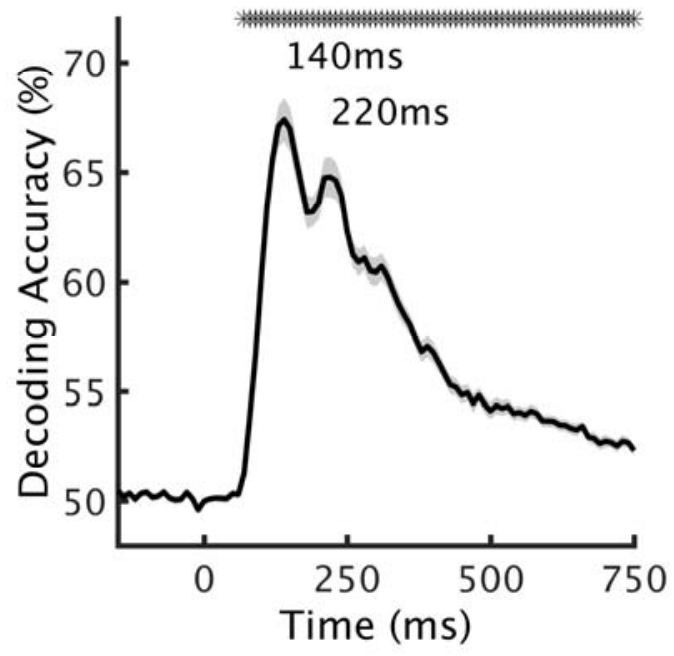

C

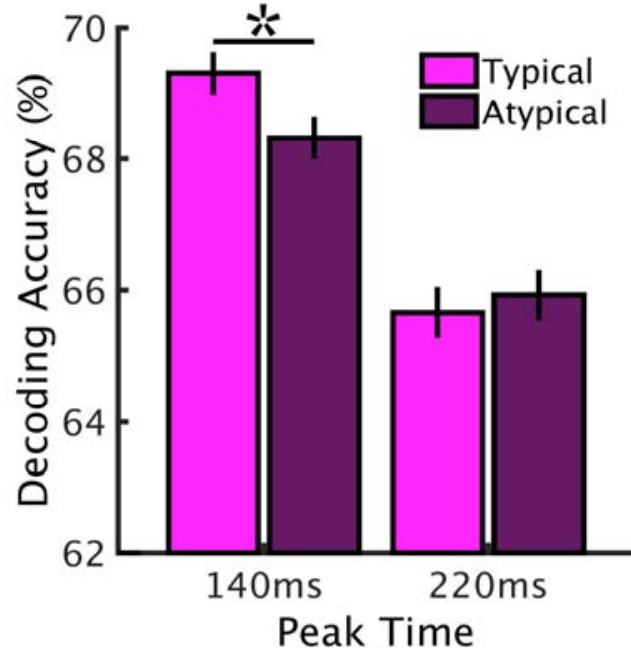

B

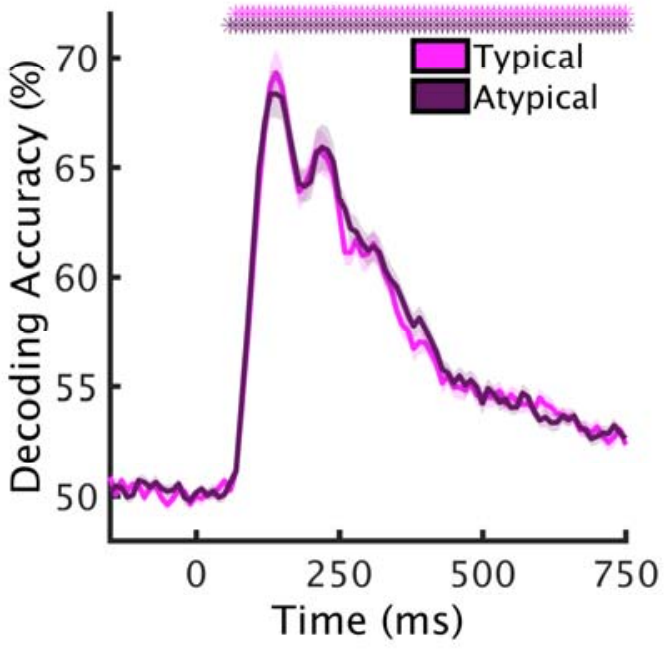

$\mathrm{E}$

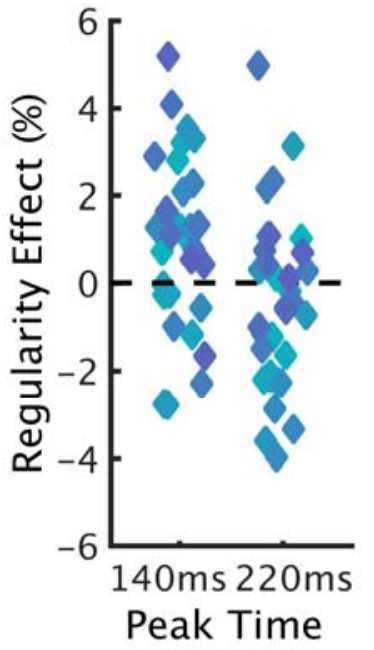

$140 \mathrm{~ms}$
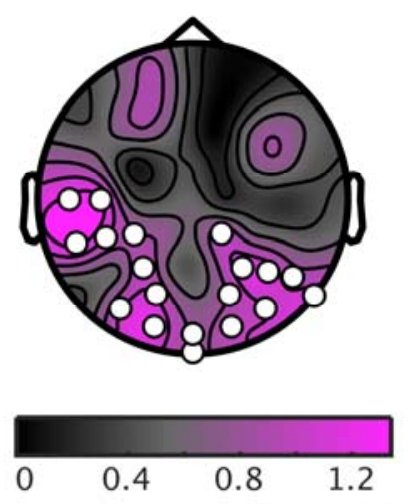

Regularity Effect (\%)

Figure 3. Classification Results. A) Overall pairwise classification performance was

313 computed by averaging all pairwise decoding time series, revealing significant decoding

314 accuracy starting at $70 \mathrm{~ms}$ after stimulus onset and peaking at $140 \mathrm{~ms}$ and $220 \mathrm{~ms}$.

315 Asterisks above data curves indicate above-chance classification ( $p<.05$, corrected for

316 multiple comparisons). The shaded margin reflects standard errors of the mean. B)

317 Classification time series for typically and atypically positioned objects were computed by averaging all pairwise classification time series comparing typically and atypically positioned objects, respectively. Classification of typically and atypically positioned pairs 
321 overall decoding. Asterisks indicate above-chance classification $(p<.05$, corrected for

322 multiple comparisons). The shaded margins reflect standard errors of the mean. C) At the

323 first decoding peak (140 ms), but not the second peak (220 ms), classification was more

324 accurate for typically than for atypically positioned objects. The asterisk indicates a

325 significant difference $(p<.05)$. Error bars reflect standard errors of the difference. D)

326 Scatterplots showing the regularity effects across participants, for both peak times. E) A

327 sensor-space classification searchlight revealed that the regularity effect (difference

328 between the classification of typically and atypically positioned objects) at the $140 \mathrm{~ms}$

329 peak is most pronounced in occipital and temporal electrodes. Circles indicate electrodes

330 exhibiting a significant regularity effect ( $p<.05$, corrected for multiple comparisons).

331

$332 \quad 3.2$ Classification of objects when positioned typically and atypically

333 To test whether neural representations differ for typically and atypically

334 positioned objects, we compared classification performance for all typically and all

335 atypically positioned objects. We averaged all pairwise classification time courses that

336 corresponded to comparisons within regular pairs (e.g., an airplane in the upper visual

337 field versus a boat in the lower visual field) and comparisons within irregular pairs (e.g.,

338 an airplane in the lower visual field versus a boat in the upper visual field) (Figure 3B). The

339 classification time series for typically and atypically positioned objects showed a similar

340 temporal structure and both replicated the peak structure observed in the overall

341 decoding, allowing for a meaningful comparison between typically and atypically

342 positioned objects at the classification peaks. We thus restricted statistical comparisons

343 to two time points of interest: the peak times observed in the overall decoding (140 ms

344 and $220 \mathrm{~ms}$ ). For the early peak at 140ms, we found higher classification accuracy for 
345 typically than for atypically positioned objects, $t(33)=3.04, p=.005, d=0.52, B F=12.06$,

346 while for the later peak at 220ms, no such difference emerged, $t(33)=0.69, p=.495, d=$

$3470.12, B F=3.37$, interaction with peak time, $F(1,33)=7.44, p=.010, \eta^{2}=0.18$ (Figure $3 C$ ).

348 This pattern of results suggests that earlier stages of object processing (as reflected in

349 the decoding peak at $140 \mathrm{~ms}$ ) benefit from typical object locations, while relatively later

350 object representations (at $220 \mathrm{~ms}$ after stimulus onset) are not sensitive to positional

351 regularities. This result was replicated in a bootstrapping analysis, where we used

352 independent sub-groups of participants to define peak times and compute the regularity

353 effects (see Supplementary Information, Fig. S1).

354 To estimate the spatial extent of the early regularity effect, we performed a

355 searchlight analysis in sensor space. We repeatedly performed the pairwise classification

356 analysis for neighborhoods of seven adjacent sensors, using only data from the early

357 peak at $140 \mathrm{~ms}$. To quantify the regularity benefit, we then computed the difference

358 between all pairwise comparisons of typically positioned objects and all pairwise

359 comparisons of atypically positioned objects at every sensor location. This analysis

360 revealed a significant regularity effect in posterior and lateral electrodes (19 significant

361 electrode sites) (Figure 3D). This result provides an indication of the tentative cortical

362 source of the regularity effect (within the limits of the restricted spatial resolution of EEG

363 measurements), suggesting that the enhanced classification for regularly positioned

364 objects may originate from visual areas of the occipital and temporal cortices.

365 These results also provide a control for oculomotor influences on our findings. If

366 differential eye-movement patterns were driving the enhanced decoding for typically

367 positioned objects, the effect should be observed in frontal electrodes, too. The absence

368 of a regularity benefit in the frontal electrodes thus provides evidence against eye- 
369 movement confounds. Two additional control analyses focused on spatiotemporal

370 response patterns in selected frontal electrodes (see Supplementary Information, Fig.

371 S3); these analyses confirmed the absence of an effect, further rendering eye-movement

372 confounds unlikely.

$373 \quad 3.3$ Classification within and between locations

374 Our classification approach collapsed across pairwise comparisons within the

375 same location and between different locations, so that classifiers could rely on

376 information from both an object's identity and its location. Therefore, the classification

377 benefit for typically positioned objects could, in principle, also emerge from a more

378 thorough coding of an object's location. For example, classifiers - in principle - only need

379 to predict the object's location to correctly classify a lamp in the upper visual field versus

380 a shoe in the lower visual field. By contrast, to correctly classify a lamp in the upper visual

381 field versus a hat in the upper visual field, classifiers need to use information about the

382 object. Note that both these comparisons contributed to the classification accuracy of

383 regularly positioned objects in the previous analysis.

384 To test whether a regularity benefit emerges also in situations where classification

385 solely depends on object information, we thus looked at the regularity effect for all

386 comparisons between locations (e.g., an airplane in the upper visual field versus a shoe in

387 the lower visual field) (Figure 4A) and all comparisons within location (e.g., an airplane in

388 the upper visual field versus a hat in the upper visual field) (Figure 4B). If typical

389 positioning enhances location information, a regularity effect should only be found when

390 comparing objects between different locations, but not within a specific location (when

391 location information is eliminated). Finding comparable effects in the within- and 
392 between-location comparisons would not support this view, indicating that typical

393 positioning can also enhance processing when only object information is diagnostic.

394 We found a main effect of visual field comparison, $F(1,33)=365.70, p<.001, \eta^{2}=$

3950.91 , with higher classification accuracies for classifying between locations (where the

396 classifier can use the stimulus' location) than within location (where the classifier has no

397 location information available), and an interaction of the within-between comparison and

398 peak latency, $F(1,33)=72.30, p<.001, \eta^{2}=0.69$, with a relatively more pronounced early

399 peak when classifying between locations. Replicating our previous results, the analysis

400 produced a significant peak $X$ regularity interaction, $F(1,33)=9.83, p=.004, \eta^{2}=0.23$, with

401 a regularity benefit at the 140 ms peak, $t(33)=3.15, p=.004, d=0.54, B F=15.52$, but not

402 the 220 ms peak, $t(33)=1.05, p=.301, d=0.18, B F=2.50$. Crucially, this pattern of results

403 did not depend on the type of classification (between locations versus within location),

$404 F(1,33)=2.53, p=.121, \eta^{2}=0.07$. These results do not provide evidence for a boost of

405 location information, thus suggesting that typical real-world locations enhance visual

406 processing by boosting object identity information. 
A

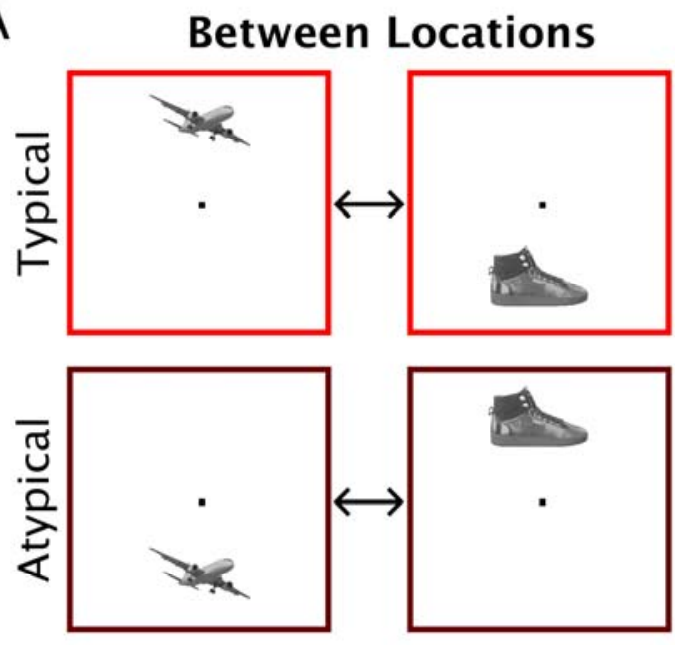

C

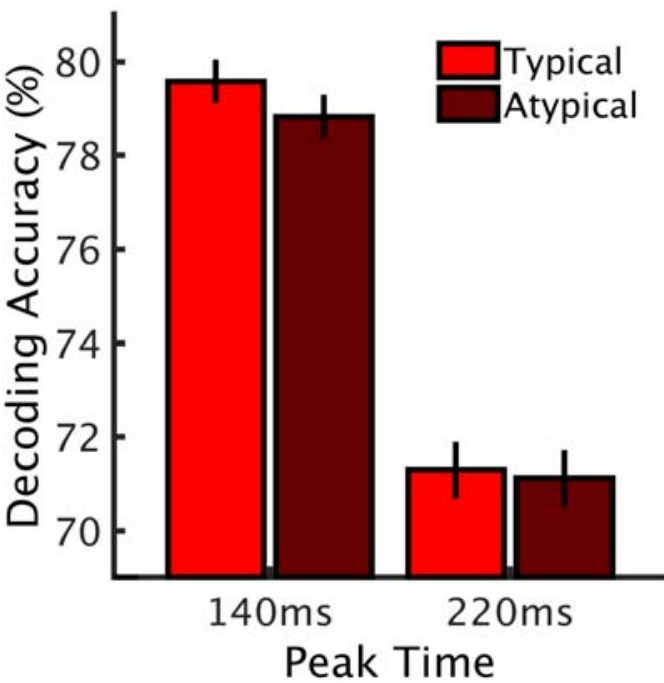

B

Within Location
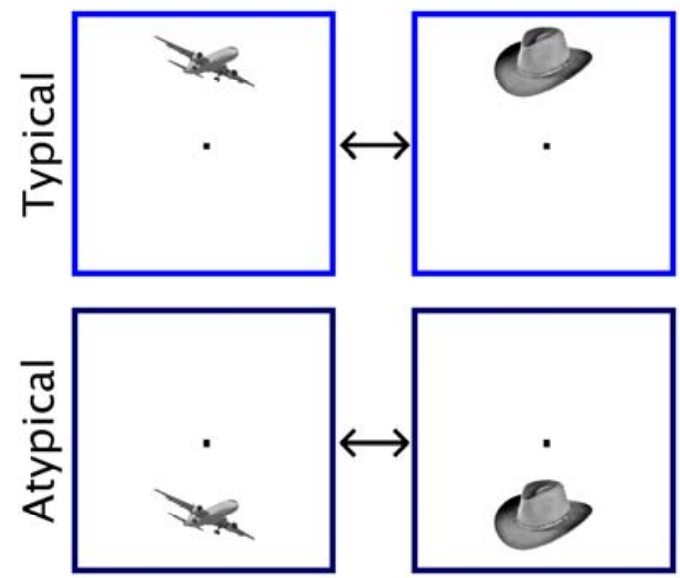

D

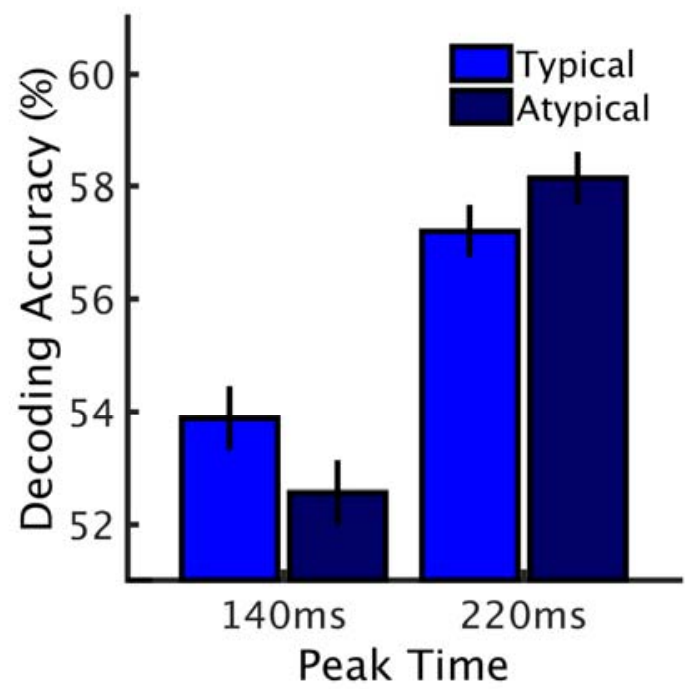

407

Figure 4. Between-Locations versus Within-Location Classification. We compared peak

decoding for typically and atypically positioned objects separately for comparisons

410 between different locations (e.g., an airplane in the upper visual field versus a shoe in the

411 lower visual field) (A) and within the same location (e.g., an airplane in the upper visual

412 field versus a hat in the upper visual field) (B). For both comparison types, we found a

413 similar pattern (C, D) with a benefit for typically positioned objects at the 140 ms peak.

414 Importantly, the patterns of results for between-locations and within-location

415 classification were statistically indistinguishable (see Results). Error bars reflect standard 


\section{Discussion}

418

420

421

422

423

424

425

\subsection{Summary}

Here, we demonstrate that positional regularities contained in real-world scenes impact brain responses to individual objects. Using multivariate classification of EEG data, we show that object coding across the visual field is affected by the typical real-world location of the object. When objects are presented in frequently experienced locations, EEG response patterns at 140 ms after stimulus onset are better discriminable than when the same objects are presented in atypical locations. This early regularity benefit yielded a robust effect size of $d=0.52$, and was consistent across subsets of participants (see Supplementary Information). Interestingly, the advantage for typically positioned objects was equally pronounced for classification between locations and within the same location, suggesting that typical positioning boosts object identity information, rather than location information. Using a sensor-space searchlight analysis, we characterize the effect in sensor space, where its most prominent emergence in posterior sensors suggests that typically positioned objects gain an advantage during early perceptual processing.

\subsection{Retinotopic priors as a consequence of natural scene structure}

How does this retinotopically specific processing benefit emerge from the structure of natural environments? An intuitively appealing explanation lies in the interplay of typical within-scene object locations and gaze patterns during natural viewing. When objects repeatedly occupy specific locations within a scene, they may repeatedly fall into similar parts of the visual field, thereby shaping visual tuning properties. For instance, as lamps mostly appear in the "upper" part of a scene, fixations likely more often land below a lamp than above a lamp. Previous eye-tracking studies can 
441 provide some evidence for such a relationship between within-scene locations and

442 retinotopic locations in limited experimental contexts: e.g., when walking along a

443 corridor, lamps most often fall in the upper visual field (Turano et al., 2003). However,

444 gaze patterns in real-world situations are complex and vary as a function of task context

445 (Hayhoe \& Ballard, 2005; Land, 2009), making it hard to establish a comprehensive

446 characterization of this relationship. Future large-scale studies that investigate viewing

447 patterns under naturalistic viewing conditions are thus required to empirically establish

448 the connection between typical within-scene locations and typical retinotopic locations

449 with high ecological validity. Without such studies, the association between typical

450 within-scene locations and retinotopic priors - although intuitively compelling - needs to

451 remain somewhat speculative.

4524.3 Early stages of object coding are sensitive to typical locations

453 Our findings demonstrate that visual processing channels are preferentially tuned

454 to specific objects appearing in specific retinotopic locations (Kaiser \& Haselhuhn, 2017;

455 Kravitz et al., 2008). Crucially, our EEG classification approach allowed us to pinpoint the

456 latency of this regularity benefit: We demonstrate that object processing at $140 \mathrm{~ms}$ after

457 stimulus onset is affected by positional regularity. The timing of the effect suggests that

458 objects appearing in typical visual field locations gain an advantage during early,

459 perceptual processing, rather than through top-down interactions (e.g., via long-range

460 feedback from frontal areas); previous M/EEG studies have suggested that such feedback

461 processes impact visual responses only at later stages, starting shortly before $200 \mathrm{~ms}$

462 (Bar et al., 2006; Fahrenfort, van Leeuwen, Olivers, \& Hogendoorn, 2017). As opposed to

463 the difference in early object processing, later representations (at $220 \mathrm{~ms}$ after stimulus

464 onset) do not depend on the location of the object. This result concurs with the 
465 increasing location tolerance over the time course of object classification, peaking at

466 around $180 \mathrm{~ms}$ (Isik et al., 2014), mirroring the increase in receptive field size along the

467 visual stream (Kravitz, Saleem, Baker, Ungerleider, \& Mishkin, 2013).

$468 \quad$ Can the sensitivity to positional regularity be interpreted as a processing

469 advantage for typically positioned objects? Or do the results rather reflect a processing

470 disadvantage for atypically positioned objects? Alternatively, there might be both an

471 experience-based advantage for typically positioned objects, and a disadvantage for

472 atypically positioned objects, caused by a lack of such experience? Our design cannot

473 conclusively disambiguate these possibilities, as it relies on relative effects (i.e.,

474 comparing typical and atypical positioning). In the Supplementary Information, we

475 however provide indirect evidence by analyzing individual-object effects: decoding of

476 typically positioned objects increases when the object has a stronger location prior, while

477 the decoding of atypically positioned objects does not vary as a function of the prior

478 strength (Fig. S2). Although these results provide tentative evidence for a processing

479 advantage for typically positioned objects (rather than a disadvantage for atypically

480 positioned objects), future investigations need to test the different interpretations more

481 explicitly, for example by including objects that are not associated with specific locations.

4824.4 Visual versus categorical sources of the regularity benefit

483 What is the content of the location-specific object representations emerging at

$484140 \mathrm{~ms}$ ? Peaks in the M/EEG decoding in this time range have been previously associated

485 with visual category processing in object-selective cortex (Cichy, Pantazis, \& Oliva, 2014,

486 2016; Carlson et al., 2013). Our searchlight analysis reveals the strongest regularity effect

487 over lateral occipital and temporal electrode sites, suggesting that the effect originates

488 from object-selective visual cortex. Whether processing differences in these object- 
489 selective regions reflect genuine category processing differences or whether they reflect

490 differential coding of category-associated visual features is a debated question (Bracci,

491 Ritchie, \& op de Beeck, 2017; Peelen \& Downing, 2017). While some data suggest that

492 visual properties explain most of the variance in object-selective responses (e.g., Baldassi

493 et al., 2013), a recent MEG decoding study has demonstrated category-selective, rather

494 than visually (shape-) driven, responses from as early as 130 ms after stimulus onset

495 (Kaiser, Azzalini, \& Peelen, 2016). To determine if the regularity benefit observed here can

496 be linked to differences in the processing of particular visual features or true categorical

497 processing differences, future studies need to employ stimuli that vary more extensively

498 in their visual characteristics.

4994.5 Positional structures beyond person perception

500 Positional regularities have been studied in humans and non-human primates

501 largely in the context of face and body perception, where parts are arranged in highly

502 predictable configurations (e.g., the features of a human face). $f M R I$ studies in humans

503 have demonstrated that individual face and body parts are processed more efficiently

504 when they appear in typical visual field locations (Chan et al., 2010; de Haas et al., 2016).

505 Single-cell recordings in monkeys demonstrated that location biases can impact cortical

506 responses to face parts as early as $100 \mathrm{~ms}$ after stimulus onset (Issa \& DiCarlo, 2012),

507 suggesting a benefit at early stages of perceptual processing.

508 Our results complement these findings by showing that such location-specific

509 object processing is not restricted to the face/body domain: The inherent structure of

510 natural scenes can similarly impact early processing (140 ms after stimulus onset) of

511 object information across the visual field. Our findings thus highlight that location-specific

512 tuning in object processing may form a general principle that shapes visual processing 
513 mechanisms for spatially predictable information. Future research could test whether

514 regularity structures also affect other domains where the visual input consists of multiple

515 parts that are constrained by spatial regularities. For example, through extensive

516 experience with reading written text, the neural mechanisms for perceiving letters could

517 get tuned to their typical spatial locations within words (Kaiser \& Haselhuhn, 2017;

518 Vinckier et al., 2007).

5194.6 Positional structures in multiple and individual objects

520 Natural environments contain positional regularities on different levels, both on

521 the levels of multiple (e.g., a lamps typically hang above tables) and individual objects

522 (e.g., a lamp is typically in the upper visual field). Previous research has primarily focused

523 on the latter: Recent behavioral studies have demonstrated that regularity structures in

524 multi-object arrangement facilitate behavior in capacity-limited visual tasks (Gronau \&

525 Shachar, 2014; Kaiser et al., 2014; Kaiser, Stein, \& Peelen, 2015; Stein, Kaiser, \& Peelen,

526 2015), and neuroimaging studies demonstrated that they enable the brain to integrate

527 information across objects that appear in frequently experienced arrangements (Baeck,

528 Wagemans, \& Op de Beeck, 2013; Kaiser \& Peelen, 2018; Kaiser et al., 2014).

529 Here, we provide the first evidence that typical regularity structures also impact

530 the neural representation of individual objects. Our finding thus raises the question

531 whether the previously reported regularity effects in multi-object perception can be

532 reduced to the effects of typical individual object location. On a behavioral level, some

533 previous studies oppose this notion by demonstrating that the benefits of multi-object

534 regularities cannot be explained by the relative location of the constituent objects (Kaiser

535 et al., 2014, 2015; Stein et al., 2015). Although these results suggest that positional

536 regularities in multi-object and single-object processing offer complementary benefits, 
537 further research is needed. Future studies will need to systematically manipulate

538 positional structures on different levels (from individual objects to multi-object

539 arrangements) to explore how regularities on multiple levels interact on a neural level.

$540 \quad 4.7$ Object processing in the context of natural scenes

541 A major challenge for the visual system when processing natural scenes is the

542 large number of individual objects they contain. Surprisingly, however, objects can be

543 rapidly decoded from MEG activity patterns, even when embedded in complex scenes

544 (Brandman \& Peelen, 2017; Kaiser, Oosterhof, \& Peelen, 2016). Previous

545 electrophysiological studies have investigated how congruent scene context can

546 facilitate cortical processing of an object. Several studies have shown that semantic

547 consistencies (i.e., whether the object is associated with the scene) affect EEG

548 waveforms starting from around 300 ms (Ganis \& Kutas, 2003; Mudrik, Lamy, \& Deouell,

549 2010; Mudrik, Shalgi, Lamy, \& Deouell, 2014; Võ \& Wolfe, 2013). More similarly to our

550 study, others have investigated the effects of syntactic consistencies (i.e., whether the

551 object is placed in its typical within-scene location), and found comparably late effects,

552 most prominently between 400 and 600 ms (Demiral, Malcolm, \& Henderson, 2012; Võ \&

553 Wolfe, 2013). Such effects of syntactic consistencies have been linked to efficient

554 behavioral performance in complex multi-object scenes (Draschkow \& Võ, 2017).

555 How can the early effect observed here be reconciled with the late effects found

556 for typical object placement within a scene? Studies on object-scene consistencies differ

557 in two important ways from our study. First, in these studies participants are cued to look

558 at the object's location within the scene, whereby retinotopic object location is

559 purposefully eliminated. Second, these studies measure overall waveform changes

560 between congruent and incongruent conditions, which may reflect more general, post- 
561 perceptual signatures of consistency processing or object-scene interaction. By contrast,

562 our analysis used highly sensitive decoding methods to explicitly focus on object

563 discriminability within typically and atypically positioned objects, so that our results are

564 best understood as a rapid signature of experience-based tuning of the visual

565 architecture. Our findings and the findings of these previous studies thus highlight

566 different facets of the processing of regularly structured environments, where both the

567 early effects of typical retinotopic locations and the later effects of typical within-scene

568 positioning may contribute to efficient real-world perception.

5694.8 Conclusion

570 Together, our results demonstrate a general principle of object coding in human

571 visual cortex: Information about an object's location and its identity are processed

572 interactively, where objects appearing in their typical retinotopic locations are more

573 efficiently coded than objects appearing in atypical retinotopic locations. This processing

574 advantage manifests in object decoding $140 \mathrm{~ms}$ after stimulus onset, suggesting that

575 early object representations are tied to the object's extensively experienced visual-field

576 location. This finding can provide a novel explanation for the efficient parsing of complex

577 real-world environments, which contain large numbers of typically positioned objects. 
bioRxiv preprint doi: https://doi.org/10.1101/177493; this version posted April 8, 2018. The copyright holder for this preprint (which was not certified by peer review) is the author/funder, who has granted bioRxiv a license to display the preprint in perpetuity. It is made available under aCC-BY-ND 4.0 International license.

\section{Acknowledgements}

580 The research was supported by a DFG Emmy-Noether Grant awarded to R.M.C. (Cl241-1/1). 


\section{$581 \quad$ References}

582 Baeck, A., Wagemans, J., \& Op de Beeck, H. P. (2013). The distributed representation of

583 random and meaningful object pairs in human occipitotemporal cortex: the

$584 \quad$ weighted average as a general rule. Neuroimage, 70, 37-47.

585

Baldassi, C., Alemi-Neissi, A., Pagan, M., DiCarlo, J. J., Zecchina, R., \& Zoccolan, D. (2013).

586

Shape similarity, better than semantic membership, accounts for the structure of

587 visual object representations in a population of monkey inferotemporal neurons. PLoS Computational Biology, 9, e1003167.

Bar, M. (2004). Visual objects in context. Nature Reviews Neuroscience, 5, 617-629.

Bar, M., Kassam, K. S., Ghuman, A. S., Boshyan, J., Schmid, A. M., Dale, A. M., Hämäläinen, facilitation of visual recognition. Proceedings of the National Academy of Sciences, U.S.A., 103, 449-454.

Biederman, I., Mezzanotte, R. J., \& Rabinowitz, J. C. (1982). Scene perception: detecting and judging objects undergoing relational violations. Cognitive Psychology, 14, 143-

Bracci, S., Ritchie, J. B., \& op de Beeck, H. P. (2017). On the partnership between neural representations of object categories and visual features in the ventral visual pathway. Neuropsychologia, 105, 153-164.

600

Brainard, D. H. (1997). The psychophysics toolbox. Spatial Vision, 10, 433-436.

601

Brandman, T., \& Peelen, M. V. (2017). Interaction between scene and object processing 
604 Carlson, T. A., Hogendoorn, H., Kanai, R., Mesik, J., \& Turret, J. (2011). High temporal

605 resolution decoding of object position and category. Journal of Vision, 11, 9.

606 Carlson, T. A., Tovar D. A., Alink, A., \& Kriegeskorte, N. (2013). Representational dynamics

607 of object vision: the first $1000 \mathrm{~ms}$. Journal of Vision, 13, 1-19.

608 Chan, A. W., Kravitz, D. J., Truong, S., Arizpe, J., \& Baker, C.I. (2010). Cortical

609 representations of bodies and faces are strongest in commonly experienced

610 configurations. Nature Neuroscience, 13, 417-418.

611 Chun, M. M. (2000). Contextual cueing of visual attention. Trends in Cognitive Sciences, 4, $612 \quad 170-178$.

613 Cichy, R. M., Chen, Y., \& Haynes, J. D. (2011). Encoding the identity and location of objects 614 in human LOC. Neuroimage, 54, 2297-2307.

615 Cichy, R. M., Pantazis, D., \& Oliva, A. (2014). Resolving human object recognition in space 616 and time. Nature Neuroscience, 17, 455-462.

617 Cichy, R. M., Pantazis, D., \& Oliva, A. (2016). Similarity-based fusion of MEG and fMRI

618 reveals spatio-temporal dynamics in human cortex during visual object

619 recognition. Cerebral Cortex, 26, 3563-3579.

620 Contini, E. W., Wardle, S. G., \& Carlson, T. A. (2017). Decoding the time-course of object

621 recognition in the human brain: From visual features to categorical decisions.

$622 \quad$ Neuropsychologia, 105, 165-176.

623 de Haas, B., Schwarzkopf, D. S., Alvarez, I., Lawson, R. P., Henriksson, L., Kriegeskorte, N., 624 \& Rees, G. (2016). Perception and processing of faces in the human brain is tuned 625 to typical facial feature locations. Journal of Neuroscience, 36, 9289-9302. 
626 Demiral, S. B., Malcolm, G. L., \& Henderson, J. M. (2012). ERP correlates of spatially incongruent object identification during scene viewing: contextual expectancy versus simultaneous processing. Neuropsychologia, 50, 1271-1285.

Draschkow, D., \& Võ, M. L.-H. (2017). Scene grammar shapes the way we interact with objects, strengthens memories, and speeds search. Scientific Reports, 7, 16471.

631 Fahrenfort, J. J., van Leeuwen, J., Olivers, C. N. L., \& Hogendoorn, H. (2017). Perceptual integration without conscious access. Proceedings of the National Academy of Sciences, U.S.A., 114, 3744-3749.

634 Ganis, G., \& Kutas, M. (2003). An electrophysiological study of scene effects on object 635 identification. Cognitive Brain Research, 16, 123-144.

636 Golomb, J. D., \& Kanwisher, N. (2012). Higher level visual cortex represents retinotopic, 637 not spatiotopic, object location. Cerebral Cortex, 22, 2794-2810.

638 Gronau, N., \& Shachar, M. (2014). Contextual integration of visual objects necessitates attention. Attention, Perception \& Psychophysics, 76, 695-714.

Hasson, U., Levy, I., Behrmann, M., Hendler, T., \& Malach, R. (2002). Eccentricity bias as an organizing principle for human high-order object areas. Neuron, 34, 479-490.

642 Hayhoe, M., \& Ballard, D. (2005). Eye movements in natural behavior. Trends in Cognitive $643 \quad$ Sciences, 9, 188-194.

644 Hemond, C. C., Kanwisher, N., \& Op de Beeck, H. P. (2007). A preference for contralateral 645 stimuli in human object- and face-selective cortex. PLoS One, 2, e574.

646 Henriksson, L., Mur, M., \& Kriegeskorte, N. (2015). Faciotopy - A face-feature like topology in the human occipital face area. Cortex, 72, 156-167. 
648 Hong, H, Yamins, D. L. K., Majaj, N. J., \& DiCarlo, J. J. (2016). Explicit information for category-orthogonal object properties increases along the ventral visual stream. Nature Neuroscience, 19, 613-622.

Isik, L., Meyers, E. M., Leibo, J. Z., \& Poggio, T. (2014). The dynamics of invariant object recognition in the human visual system. Journal of Neurophysiology, 111, 91-102.

Issa, E. B., \& DiCarlo, J. J. (2012). Precedence of the eye region in neural processing of faces. Journal of Neuroscience, 32, 16666-16682.

Kaiser, D., Azzalini, D. C., \& Peelen, M. V. (2016). Shape-independent object category responses revealed by MEG and fMRI decoding. Journal of Neurophysiology, 115, $2246-2250$.

Kaiser, D., \& Haselhuhn, T. (2017). Facing a regular world: How spatial object structure shapes visual processing. Journal of Neuroscience, 37, 1965-1967.

Kaiser, D., Oosterhof, N. N., \& Peelen, M. V. (2016). The neural dynamics of attentional 661 selection in natural scenes. Journal of Neuroscience, 36, 10522-10528.

Kaiser. D., \& Peelen, M. V. (2018). Transformation from independent to integrative coding of multi-object arrangements in human visual cortex. Neuroimage, 169, 334-341.

664 Kaiser, D., Stein, T., \& Peelen, M. V. (2014). Object grouping based on real-world regularities facilitates perception by reducing competitive interactions in visual cortex. Proceedings of the National Academy of Sciences, U.S.A., 111, 11217-11222.

667 Kaiser, D., Stein, T., \& Peelen, M. V. (2015). Real-world spatial regularities affect visual working memory for objects. Psychonomic Bulletin \& Review, 22, 1784-1790.

669 Kravitz, D. J., Kriegeskorte, N., \& Baker, C. I. (2010). High-level visual object representations are constrained by position. Cerebral Cortex, 20, 2916-2925. 
671 Kravitz, D. J., Saleem, K. S., Baker, C. I., Ungerleider, L. G., \& Mishkin, M. (2013). The 672 ventral visual pathway: an expanded neural framework for the processing of 673 object quality. Trends in Cognitive Sciences, 17, 26-49.

674 Kravitz, D. J., Vinson, L. D., \& Baker, C. I. (2008). How position dependent is visual object 675 recognition? Trends in Cognitive Sciences, 12, 114-122.

676 Land, M. F. (2009). Vision, eye movements, and natural behavior. Visual Neuroscience, 26, $677 \quad 51-62$.

678 Mudrik, L., Lamy, D., \& Deouell, L. Y. (2010). ERP evidence for context congruity effects 679 during simultaneous object-scene processing. Neuropsychologia, 48, 507-517.

680 Mudrik, L., Shalgi, L., Lamy, D., \& Deouell, L. Y. (2010). ERP correlates of spatially 681 incongruent object identification during scene viewing: contextual expectancy 682 versus simultaneous processing. Neuropsychologia, 56, 447-458.

683 Oliva, A., \& Torralba, A. (2007). The role of context in object recognition. Trends in 684 Cognitive Sciences, 11, 520-527.

685 Oostenveld, R., Fries, P., Maris, E., \& Schoffelen, J. M. (2011). Fieldtrip: open source 686 software for advances analysis of MEG, EEG, and invasive electrophysiological data. Computational Intelligence and Neuroscience, 2011, 156869.

688 Oosterhof, N. N., Connolly, A. C., \& Haxby, J. V. (2016). CoSMoMVPA: multi-modal multivariate pattern analysis of neuroimaging data in Matlab / GNU Octave. Frontiers in Neuroinformatics, 10, 20.

Peelen, M. V., \& Downing, P. E. (2017) Category selectivity in human visual cortex: Beyond visual object recognition. Neuropsychologia, 105, 177-183.

693 Poldrack, R. A. (2007). Region of interest analysis for fMRI. Social Cognitive and Affective Neuroscience, 2, 67-70. 
695 Rouder, J. N., Speckman, P. L., Sun, D., Morey, R. D., \& Iverson, G. (2009). Bayesian t-tests

696 for accepting and rejecting the null hypothesis. Psychonomic Bulletin \& Review, 16,

$697 \quad 225-237$.

698 Russell, B. C., Torralba, A., Murphy, K. P., \& Freeman, W. T. (2008). LabelMe: a database

699 and web-based tool for image annotation. International Journal of Computer Vision,

$700 \quad 77,157-173$

701 Schwarzlose, R. F., Swisher, J. D., Dang, S., \& Kanwisher, N. (2008). The distribution of

702 category and location information across object-selective regions in human visual

703 cortex. Proceedings of the National Academy of Sciences, U.S.A., 105, 4447-4452.

704 Smith, S. M., \& Nichols, T. E. (2009). Threshold-free cluster enhancement: addressing

705 problems of smoothing, threshold dependence and localization in cluster

706 interference. Neuroimage, 66, 215-222.

707 Stein, T., Kaiser, D., \& Peelen, M. V. (2015). Interobject grouping facilitates visual

708 awareness. Journal of Vision, 15, 10.

709 Turano, K. A., Geruschat, D. R., \& Baker, F. H. (2003). Oculomotor strategies for the

710 direction of gaze tested with a real-world activity. Vision Research, 43, 333-346.

711 Vinckier, F., Dehaene, S., Jobert, A., Dubus, J. P., Sigman, M., \& Cohen, L. (2007).

712 Hierarchical coding of letter strings in the ventral stream: dissecting the inner

713 organization of the visual word-form system. Neuron, 55, 143-156.

714 Võ, M. L.-H., \& Wolfe, J. M. (2013). Differential ERP signatures elicited by semantic and

715 syntactic processing in scenes. Psychological Science, 24, 1816-1823.

716 Willenbockel, V., Sadr, J., Fiset, D., Horne, G. O., Gosselin, F., \& Tanaka, J. W. (2010).

717 Controlling low-level image properties: The SHINE toolbox. Behavior Research

$718 \quad$ Methods, 42, 671-684. 
bioRxiv preprint doi: https://doi.org/10.1101/177493; this version posted April 8, 2018. The copyright holder for this preprint (which was not certified by peer review) is the author/funder, who has granted bioRxiv a license to display the preprint in perpetuity. It is made available under aCC-BY-ND 4.0 International license.

719 Wolfe, J. M., Võ, M. L.-H., Evans, K. K., \& Greene, M. R. (2011). Visual search in scenes

720 involves selective and nonselective pathways. Trends in Cognitive Sciences, 15, 77-

721 84. 